\title{
Maximum and Minimum Transmittance of a Structure Containing N-Identical Pairs of Left- and Right-Handed Materials
}

\author{
Muin F. Ubeid1,*, Mohammed M. Shabat ${ }^{1}$, Mohammed O. Sid-Ahmed ${ }^{2}$ \\ ${ }_{1}^{1}$ Department of Physics, Faculty of Science, Islamic University of Gaza, P.O.Box 108, Gaza, \\ Gaza Strip, Palestinian Authority \\ 2 Department of Physics, Faculty of Science, Sudan University of Science and Technology, \\ The Republic of The Sudan
}

(Received 23 July 2012; revised manuscript received 16 November 2012; published online 29 December 2012)

\begin{abstract}
The optical transmission properties of a structure consisting of $\mathrm{N}$ identical pairs of left- and rightmaterials are investigated theoretically and numerically. Maxwell's equations are used to determine the electric and magnetic fields of the incident waves at each layer. Snell's law is applied and the boundary conditions are imposed at each layer interface to calculate Fresnel coefficients. Expressions for reflectance and transmittance of the structure are given in terms of these coefficients. In the numerical results the transmittance of the structure is computed and illustrated as a function of frequency under different values of N. Minimum transmittance is achieved by using high and low opposite refractive indices of left and right materials of each pair of the structure. The frequency band of this transmittance is reduced by decreasing N. Maximum transmittance is demonstrated by using two slabs of the same width and opposite refractive indices placed between two dielectric media of the same kind. The effect of frequency and angle of incidence is very weak in these structures as compared to their all-dielectric counterparts. Moreover the obtained results are in agreement with the law of conservation of energy.
\end{abstract}

Keywords: Angle of incidence, Electromagnetic waves, Frequency, Left-handed material, Refractive index, Reflectance, Transmittance.

PACS numbers: 42.25Bs, 42.25.Dd, 42.25.Gy

\section{INTRODUCTION}

Metamaterials (sometimes termed left-handed (LH) materials) are materials whose permittivity $\varepsilon$ and permeability $\mu$ are both negative and consequently have negative index of refraction. These materials are artificial and theoretically discussed first by Veselago [1] over 40 years ago. The first realization of such materials, consisting of split-ring resonators (SRRs) and continuous wires, was first introduced by Pendry $[2,3]$. Regular materials are materials whose $\varepsilon$ and $\mu$ are both positive and termed right handed $(\mathrm{RH})$ materials. R.A. Shelby et al. [4] have studied negative refraction in LH materials. Kong [5] has provided a general formulation for the electromagnetic wave interaction with stratified metamaterial structures. M.F. Ubeid et al. [6] have presented a numerical study of a structure containing lefthanded material waveguide. R.W. Ziolkowski et al. [7] haves studied wave propagation in media having negative permittivity and permeability. N. Enghheta et al. [8] have shown some interesting applications of $\mathrm{LH}$ material. C. Sabah [9] has investigated the effects of loss factor on plane wave propagation through a lefthanded material slab. H. Oraiz et al. [10] have formulated a theory for zero reflection of electromagnetic waves from multilayered metamaterial structures. P. Pereyra et al. [11] have studied the effect of complex and negative indices in the transmission of electromagnetic waves through superlattices. C. Sabah et al. [12] have obtained theoretically high reflection coating with negative and positive refractive indices. H. Cory et al. [13] have analyzed the reflection and transmission characteristics of a multilayered structure consisting of left-handed materials and dielectric slabs.

This paper is interested in the transmission properties of a structure consisting of $\mathrm{N}$ identical pairs of leftand right-handed media (LH-RH media). Maximum and minimum transmittance of the structure are demonstrated on the rule that, LH material has negative index of refraction while $\mathrm{RH}$ material has positive index of refraction. Minimum transmittance is obtained by using high ratio of refractive indices and different thicknesses of $\mathrm{LH}$ and $\mathrm{RH}$ media constituted each pair of the structure. The total thickness of each pair is quarter wavelength of the incident waves at the operation frequency. The frequency band of minimum transmittance is affected by the number of LH-RH media pairs. Maximum transmittance is formed by two slabs of the same thickness and opposite refractive indices, one is $\mathrm{LH}$ and the other is $\mathrm{RH}$ material. The slabs are situated between two media of the same kind like vacuum. It is observed that the frequency and angle of incidence are much less dominant in these configurations than in their all-dielectric media. In addition the transmittance-frequency curves show no ripples and a monotonous quasi symmetric rise to the left and to the right of the central frequency. In the theory the electric and magnetic fields of the incident waves are calculated at each layer using Maxwell's equations. The boundary conditions are imposed at each interface and Snell's law is used to obtain the reflection and transmission coefficients in terms of Fresnel coefficients. The overall reflectance and transmittance of the structure are formulated in terms of these coefficients. A recursive method is applied on the computations under the mentioned conditions to show the variation of

\footnotetext{
*mubeid@mai.iugaza.edu
} 
transmittance with frequency. To check the results of the analysis, it is found that the conservation of energy is satisfied for all computations.

\section{THEORY}

Consider a pair of LH $\left(-\varepsilon 2,-\mu_{0}\right)$ and $\mathrm{RH}\left(\varepsilon_{3}, \mu_{0}\right)$ materials is situated between two semi-finite dielectric media. A perpendicular polarized plane wave in region 1 is incident on the plane $z=0$ at some angle $\theta$ relative to the normal to the boundary (see Fig. 1).

The electric field in each region is $[14,15]$ :

$$
\vec{E}_{\ell}=\left(A_{\ell} e^{i k_{t z} z}+B_{\ell} e^{-i k_{t z} z}\right) e^{i\left(k_{t x} x-\omega t\right)} \hat{y}
$$

To find the corresponding magnetic field $\vec{H}_{\ell}$, we start with Maxwell's equation $\vec{\nabla} \times \vec{E}_{\ell}=-\frac{\partial \vec{B}}{\partial t}$, substituting $\vec{B}=\mu_{\ell} \vec{H}_{\ell}$ and solving for $\vec{H}_{\ell}$ yield:

$$
\vec{H}_{\ell}=\frac{1}{\mu_{\ell} \omega}\left[\begin{array}{l}
\left(A_{\ell} k_{\ell x} e^{i k_{\ell z} z}+B_{\ell} k_{\ell x} e^{-i k_{\ell z} z}\right) \hat{z} \\
+\left(-A_{\ell} k_{\ell z} e^{i k_{\ell z} z}+B_{\ell} k_{\ell z} e^{-i k_{\ell z} z}\right) \hat{x}
\end{array}\right] e^{i\left(k_{\ell x} x-\omega t\right)}
$$

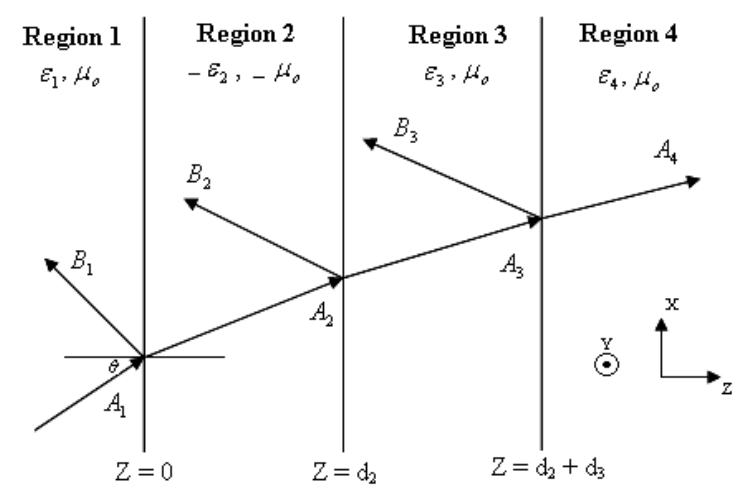

Fig. 1 - Wave propagation through a structure consisting of a pair of dielectric and metamaterial embedded between two dielectric semi-infinite media

Where $A_{l}$ and $B_{l}$ are the amplitude of forward and backward travelling waves $(l=1,2,3,4), k_{l}=n_{l} \omega / c$ is the wave vector inside the material and $n_{l}$ is the refractive index of it.

Matching the boundary conditions for $\vec{E}$ and $\vec{H}$ fields at each layer interface, that is at $z=0, E_{1 y}=E_{2 y}$ and $H_{1 y}=H_{2 y}$ and so on. This yields six equations with six unknown parameters $[14,16,17]$ :

$$
\begin{gathered}
A_{1}+B_{1}=A_{2}+B_{2} \\
\frac{k_{1 z}}{\mu_{1}}\left(A_{1}-B_{1}\right)=\frac{k_{2 z}}{\mu_{2}}\left(A_{2}-B_{2}\right) \\
A_{2} e^{i k_{2_{2}} d_{2}}+B_{2} e^{-i k_{2 z} d_{2}}=A_{3} e^{i k_{3 z} d_{2}}+B_{3} e^{-i k_{3 z} d_{2}} \\
\frac{k_{2 z}}{\mu_{2}}\left(A_{2} e^{i k_{2 z} d_{2}}-B_{2} e^{-i k_{2 z} d_{2}}\right)=\frac{k_{3 z}}{\mu_{3}}\left(A_{3} e^{i k_{3 z} d_{2}}-B_{3} e^{-i k_{3 z} d_{2}}\right) \\
A_{3} e^{i k_{3 z}\left(d_{2}+d_{3}\right)}+B_{3} e^{-i k_{3 z}\left(d_{2}+d_{3}\right)}=A_{4} e^{i k_{4 z}\left(d_{2}+d_{3}\right)}
\end{gathered}
$$

$$
\frac{k_{3 z}}{\mu_{3}}\left(A_{3} e^{i k_{3 z}\left(d_{2}+d_{3}\right)}-B_{3} e^{-i k_{3 z}\left(d_{2}+d_{3}\right)}\right)=\frac{k_{4 z}}{\mu_{4}} A_{4} e^{i k_{4 z}\left(d_{2}+d_{3}\right)}
$$

Where $k_{1 x}=k_{2 x}=k_{3 x}=k_{4 x} \equiv$ Snell's law and:

$$
k_{\ell z}=\frac{\omega}{c} \sqrt{n_{\ell}^{2}-n_{1}^{2} \sin ^{2} \theta}
$$

Fresnel coefficients (interface reflection and transmission coefficients $r, t$ respectively) for perpendicular polarized light are given by [18]:

$$
\begin{aligned}
& r_{i j}=\frac{\mu_{j} k_{i z}-\mu_{i} k_{j z}}{\mu_{j} k_{i z}+\mu_{i} k_{j z}} \\
& t_{i j}=\frac{2 \mu_{j} k_{i z}}{\mu_{j} k_{i z}+\mu_{i} k_{j z}}
\end{aligned}
$$

Where $i, j$ correspond to any two adjacent media, $\mu_{\ell}=-\left|\mu_{\circ}\right|, \quad k_{\ell z}=-\left|k_{\ell z}\right|$ for LH medium and $\mu_{\ell}=+\left|\mu_{\circ}\right|$, $k_{\ell z}=+\left|k_{\ell z}\right|$ for RH medium $(l=i, j)[1,14,18]$.

The reflection and transmission coefficients $R$ and $T$ respectively of the structure are five by [5]:

$$
\begin{aligned}
& R=\frac{B_{1}}{A_{1}}=\frac{r_{12}+r_{12} r_{23} r_{34} e^{i 2 k_{3 z} d_{3}}+r_{23} e^{i 2 k_{2 z} d_{2}}+r_{34} e^{i 2\left(k_{2 z} d_{2}+k_{3 z} d_{3}\right)}}{1+r_{23} r_{34} e^{i 2 k_{3 z} d_{3}}+r_{12} r_{23} e^{i 2 k_{22} d_{2}}+r_{12} r_{34} e^{i 2\left(k_{22} d_{2}+k_{3 z} d_{3}\right)}} \\
& T=\frac{A_{4}}{A_{1}}=\frac{t_{12} t_{23} t_{34} e^{i\left(k_{2 z} d_{2}+k_{32} d_{3}\right)}}{1+r_{23} r_{34} e^{i 2 k_{32} d_{3}}+r_{12} r_{23} e^{i 2 k_{22} d_{2}}+r_{12} r_{34} e^{i 2\left(k_{2 z} d_{2}+k_{32} d_{3}\right)}}
\end{aligned}
$$

The reflectance $R^{\prime}$ and transmittance $T^{\prime}$ of the structure are given by $[14,15,19,20]$ :

$$
R^{\prime}=R R^{*}, T^{\prime}=\frac{k_{4 z}}{k_{1 z}} T T^{*}
$$

Where $R^{*}$ and $T^{*}$ are the complex conjugate of $R$ and $T$ respectively. The law of conservation of energy is given by [5]:

$$
R^{\prime}+T^{\prime}=1
$$

For $n$-layers structure shown in Fig. $2 R$ and $T$ are calculated as follows [5, 21]:

$$
\begin{array}{l|l|l|l|l|l|}
2 & 3 & & n-2 & n-1 & n \\
& & \ldots & & &
\end{array}
$$

Fig. $2-n$-layers of different thicknesses and refractive indices are embedded between two dielectric media

$$
\begin{gathered}
R_{n}=r_{n-1, n} \\
R_{n-1}=\frac{r_{n-2, n-1}+R_{n} e^{i 2 k_{(n-1) z} d_{n-1}}}{1+r_{n-2, n-1} R_{n} e^{i 2 k_{(n-1) z} d_{n-1}}}
\end{gathered}
$$




$$
R_{n-2}=\frac{r_{n-3, n-2}+R_{n-1} e^{i 2 k_{(n-2) z} d_{n-2}}}{1+r_{n-3, n-2} R_{n-1} e^{i 2 k_{(n-2) z} d_{n-2}}}
$$

Continue on the same procedure until $R_{2}$ is reached which is the reflectance of the structure as a whole.

$$
R_{2}=\frac{r_{12}+R_{3} e^{i 2 k_{2 z} d_{2}}}{1+r_{12} R_{3} e^{i 2 k_{2 z} d_{2}}}
$$

The same procedure is performed for $T_{2}$ :

$$
\begin{gathered}
T_{n}=t_{n-1, n} \\
T_{n-1}=\frac{t_{n-2, n-1} T_{n} e^{i k_{(n-1) z} d_{n-1}}}{1+r_{n-2, n-1} R_{n} e^{i 2 k_{(n-1) z} d_{n-1}}} \\
T_{n-2}=\frac{t_{n-3, n-2} T_{n-1} e^{i k_{(n-2) z} d_{n-2}}}{1+r_{n-3, n-2} R_{n-1} e^{i 2 k_{(n-2) z} d_{n-2}}} \\
T_{2}=\frac{t_{12} T_{3} e^{i k_{2 z} d_{2}}}{1+r_{12} R_{3} e^{i 2 k_{2 z} d_{2}}}
\end{gathered}
$$

Where $d_{2}, d_{n-1}$ and $d_{n-2}$ are thicknesses of layers 2 , $n-1$ and $n-2$ respectively.

Maximum Transmittance: In order to minimize $R$ and maximize $T$, a pair of slabs of $\mathrm{LH}$ and $\mathrm{RH}$ materials of the same width and opposite refractive indices are situated between two semi-infinite dielectric media of the same kind. By referring to Fig. 1 and under these conditions, $\theta_{2}=\theta_{3}, k_{2 z}=-\left|k_{3 z}\right|, r_{23}=0, t_{23}=1, r_{12}=-r_{34}$, $t_{12} t_{34}=1+r_{12} r_{34}, e^{i 2\left(k_{2 z} d_{2}+k_{3 z} d_{3}\right)}=1$. Consider these relations into eqs. $(12,13)$ yields $R=0, T=1$ for any frequency and for any angle of incidence.

Minimum Transmittance: In order to maximize $\mathrm{R}$ and minimize $\mathrm{T}$, the relation between refractive indices and widths of the slabs are such that: $k_{2 z} d_{2}=-\left|k_{3 z} d_{3}\right|=-\pi / 2$ at the central frequency $\omega_{0}$ for normal incidence $\theta=0$ (this is the first condition), and: $n_{1}<\left|n_{2}\right|,\left|n_{2}\right|>n_{3}, n_{3}<n_{4}$ (this is the second condition).

From the first condition: $n_{2} d_{2}=-\left|n_{3} d_{3}\right|=-c \pi / 2 \omega$ 。 at any frequency $\omega^{\prime}$ :

$$
\begin{gathered}
k_{2 z} d_{2}=-\frac{\omega^{\prime}}{\omega_{\circ}} \frac{\pi}{2} \cos \theta_{2} \\
k_{3 z} d_{3}=\frac{\omega^{\prime}}{\omega_{\circ}} \frac{\pi}{2} \cos \theta_{3} \\
i 2\left(k_{3 z} d_{3}+k_{2 z} d_{2}\right)=i \pi \frac{\omega^{\prime}}{\omega_{\circ}}\left(\cos \theta_{3}-\cos \theta_{2}\right)
\end{gathered}
$$

For dielectric adjacent to dielectric, the negative sign in the last equation is replaced by positive sign. It is clear that $\left(\cos \theta_{3}-\cos \theta_{2}\right)$ is smaller than $\left(\cos \theta_{3}+\cos \theta_{2}\right)$.

From the second condition $\theta_{2}$ and $\theta_{3}$ are very small. This can be realized from Snell's law: $\sin \theta_{2}=\sin \theta_{1} n_{1} / n_{2}$, $\sin \theta_{3}=\sin \theta_{2} n_{2} / n_{3}$, in this case:

$$
k_{2 z} d_{2} \approx-\pi / 2, k_{3 z} d_{3} \approx \pi / 2, i 2\left(k_{3 z} d_{3}+k_{2 z} d_{2}\right) \approx 0
$$

Applying the last two conditions and their results on eqs. $(12,13)$ yield: $r_{12}<0, r_{23}>0, r_{34}<0, r_{12} r_{23} r_{34}>0$, $e^{i 2 k_{22} d_{2}}=e^{i 2 k_{32} d_{3}} \approx-1, \quad e^{i 2\left(k_{22} d_{2}+k_{3 z} d_{3}\right)} \approx 1, \quad t_{12}>0, \quad t_{23}>0$, $t_{34}>0$. By comparing eqs. $(12,13)$, it is noticed that the denominator is the same. The numerator of eq. (13) becomes smaller since $t_{12}, t_{23}, t_{34}$ are multiplied by each other and each of them is less than unity. Therefore $T$ becomes very small. While each term in the numerator of eq. (12) is negative and the sum of them does not cancel out. Consequently the value $R^{\prime}$ becomes larger.

\section{NUMERICAL RESULTS AND DISCUSSION}

In calculations, a perpendicular polarization of the incident waves is considered and the operating frequency is selected to be $f_{0}=20 \mathrm{GHz}$. The thickness of each pair of the LH and RH slabs is quarter wavelength at the operation frequency.

Maximum transmittance: Fig. 3 shows the transmittance of the structure considered in Fig. 1 as a function of frequency for three angles of incidence $\left(\theta=0^{\circ}\right.$, $\left.\theta=20^{\circ}, \theta=40^{\circ}\right)$. Region 1 and Region 4 are air. The LH and RH slabs are of the same thickness $\left(d_{2}=d_{3}=\lambda / 8\right)$ and opposite refractive indices, that of $\mathrm{RH}$ material is selected to be 1.434 (fluorine) and that of LH material is -1.434 . As seen from the figure, when the conditions of maximum transmittance are satisfied, the transmittance is always equal to unity for any frequency and for any angle of incidence.

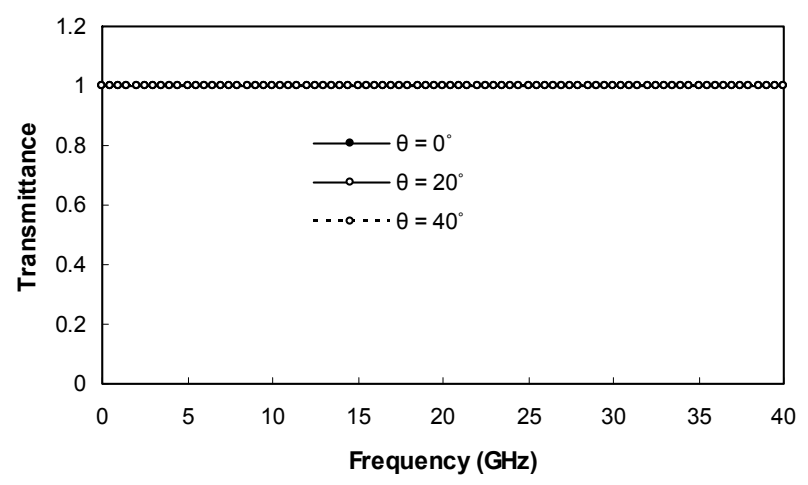

Fig. 3 - The transmittance as a function of frequency of a pair of slabs of $\mathrm{LH}$ and $\mathrm{RH}$ materials embedded in air. The slabs have the same thickness and opposite refractive indices

Minimum transmittance: In order to illustrate the effect of LH material on the minimization of the transmittance, the conditions of minimum transmittance are first applied on a structure of all dielectric pairs. Figure 4 points out the transmittance as a function of frequency for all dielectric-pairs structure for three angles of incidence $\left(\theta=0^{\circ}, \theta=20^{\circ}, \theta=40^{\circ}\right)$. The structure has ten identical pairs of dielectrics situated in air. The refractive indices of each pair of dielectrics are selected to be 3.5 (gallium phosphide) and 1.434 (fluorite), this satisfies the second condition. The thickness of the higher refractive index slab is $0.00107 \mathrm{~m}$ and that of the lower one is $0.00262 \mathrm{~m}$. These values are chosen such that, the first condition of minimum transmittance is satisfied and the total length of each pair of slabs is 
$\lambda / 4$ at the operation frequency. As observed from the figure, the transmittance becomes zero at and around the central frequency. The symmetry shifts in frequency to the right side when the incident angle increased. Also there is more ripples in the frequency behavior to the left and to the right of zero transmittance.

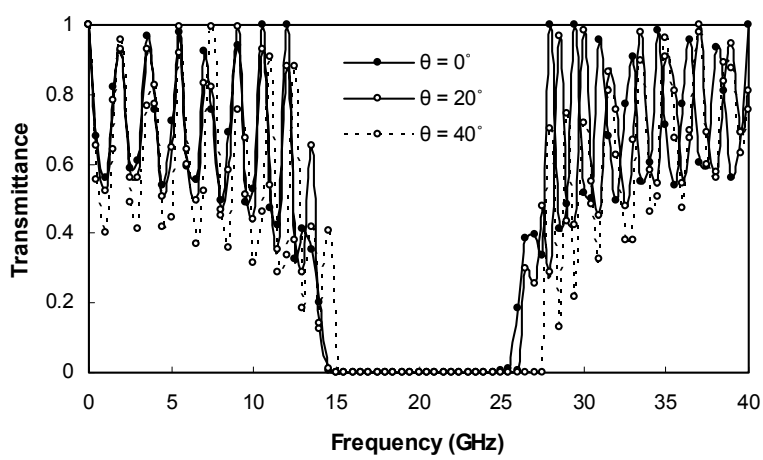

Fig. 4 - Transmittance for all dielectric structure as a function of frequency for various angles of incidence

Fig. 5 presents the transmittance versus frequency at the incidence angle of $20^{\circ}$ for three numbers of identical LH-RH pairs structure embedded in air $(N=4$, $N=7, N=10$ ) (where $N$ is the number of LH-RH pair and $n$ is the number of layers $n=2 N+2$ ). The refractive index and thickness are $-1.434,0.00262 \mathrm{~m}$ for the $\mathrm{LH}$ material and $3.5,0.00107 \mathrm{~m}$ for the $\mathrm{RH}$ material. The total length of each LH-RH pair is $\lambda / 4$ at the operation frequency and the two conditions for minimum transmittance are satisfied by these values. From the figure it is seen that, the frequency band of zero transmittance is larger as compared to all dielectric structures. The mentioned band is reduced by decreasing the value of $N$. In addition the LH-RH-pairs structure show no ripples and a monotonous quasi symmetric arise to the left and to the right of the central frequency.

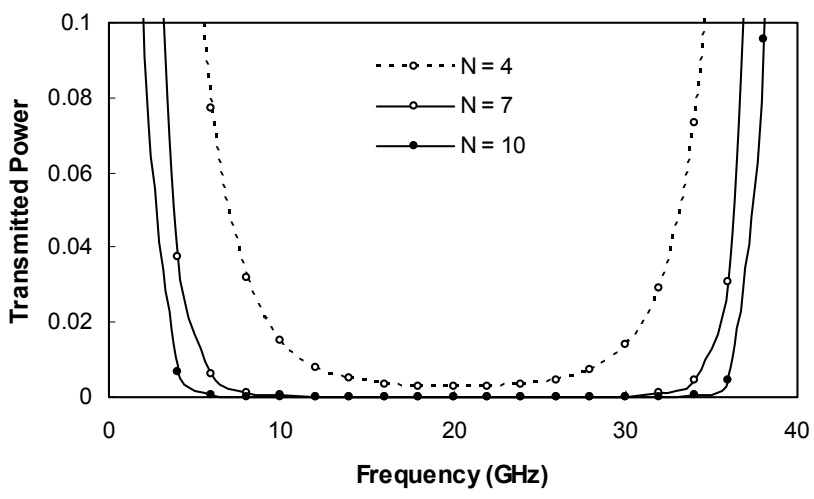

Fig. 5 - Transmittance for LH-RH structure as a function of frequency for various numbers of LH-RH pairs

Fig. 6 depicts the transmittance against frequency for three angles of incidence $\left(\theta=0^{\circ}, \theta=20^{\circ}, \theta=40^{\circ}\right)$ and for $N=10$. The other parameters which satisfy the two conditions of minimum transmittance are kept the same as in Fig. 5. As confirmed from the figure, the influence of the angle of incidence is very weak as compared to the all dielectric structures.

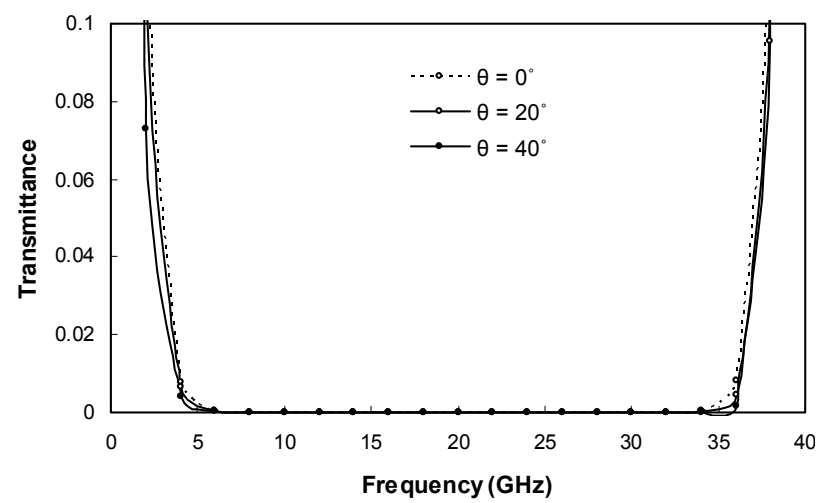

Fig. 6 - Reflectance and Transmittance for LH-RH structure against frequency for various angles of incidence

Fig. 7 illustrates the transmittance-frequency variation when the first condition of minimum transmittance does not satisfied $\left(n_{2} d_{2} \neq n_{3} d_{3}\right)$. The number of $\mathrm{LH}-\mathrm{RH}$ pairs is ten, the LH material is fluorite (thickness $4 \lambda$ ) and the $\mathrm{RH}$ material is air (thickness $2 \lambda$ ), where $\lambda$ is calculated at the operation frequency.

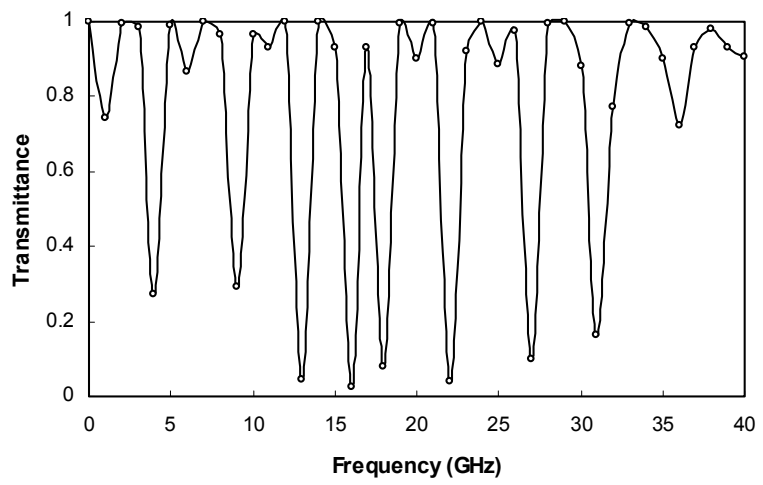

Fig. 7 - Transmittance for LH-RH structure as a function of frequency when the first condition of minimum transmittance does not satisfied

\section{CONCLUSIONS}

Regarding with the transmittance, the behavior of LH-RH materials is different from $\mathrm{RH}-\mathrm{RH}$ materials. Maximum transmittance has been formed by placing one pair of $\mathrm{LH}$ and $\mathrm{RH}$ slabs in air. The slabs have the same width and opposite refractive indices. Minimum transmittance has been demonstrated by placing a number of identical LH-RH pairs of slabs in air. The slabs of each pair have high and low opposite refractive indices and different thicknesses. It has been found that for LH-RH structures the frequency band of zero transmittance is larger as compared to all dielectric structure and the mentioned band is reduced by decreasing the number of LH-RH pairs. Furthermore, these structures display no ripples but a monotonous quasi symmetric in the transmittance to the left and to the right of operation frequency is observed. The discussed problem gives rise to possibilities of tuning the propagation for applications in antenna radome, microwave, millimeter wave and optical devices. 


\section{REFERENCES}

1. V.G. Veselago, Sov. Phys. Usp. 10, 509 (1968).

2. J.B. Pendry, A.J. Holden, W.J. Sewart, I. Youngs, Phys. Rev. Lett. 76, 4773 (1996).

3. J.B. Pendry A.J. Holden, D.J. Robbins, W.J. Stewart, IEEE T. Microw. Theory 47, 2075 (1999).

4. R.A. Shelby, D.R. Smith, S. Schultz, Science 292, 77 (2001).

5. J.A. Kong, Prog. Electromagn. Res. 35, 1 (2002).

6. M.F. Ubeid, M.M. Shabat, M.O. Sid-Ahmed, Indian J. Phys. 86125 (2012).

7. R.W. Ziolkowski, E. Heyman, Phys. Rev. E 64, 056625 (2001)

8. N. Enghheta, R.W. Ziolkowski, IEEE T. Microw. Theory 53, 1535 (2005).

9. C. Sabah, Acta Phys. Pol. A 113, 1589 (2008).

10. H. Oraizi, A. Abdolali, IET Microw. Antenna. P. 3, 987 (2009).

11. P. Pereyra, A. Robledo-Martinez, M. Morales-Luna, Microelectron. J. 39, 394 (2008).

12. C. Sabah, S. Uckun, Piers Online 5, 601 (2009).

13. H. Cory, C. Zach, Microw. Opt. Techn. Let. 40, 460 (2004).
14. R.A. Shelby, Microwave Experiments with Left-Handed Materials, (University of California: San Diego: 2001)

15. J.A. Kong, Theory of Electromagnetic waves (EMW Publishing: 2005).

16. M.F. Ubeid, M.M. Shabat, M.O. Sid-Ahmed, Natural Science 3, 328 (2011)

17. M.F. Ubeid, M.M. Shabat, M.O. Sid-Ahmed, J. Nano- Electron. Phys. 4 No. 1, 01009(1-4) (2012).

18. C. Caloz, T. Itoh, Electromagnetic metamaterials: Transmission Line Theory and Microwave Applications (New Jersey: John Wiley and sons: 2006).

19. M.F. Ubeid, M.M. Shabat, International Conference on Advanced Optoelectronics and Lasers (CAOL), 137 (Ukraine: Sevastopol: 2010).

20. M.F. Ubeid, M.M. Shabat, International Kharkiv Symposium on Physics and Engineering of Microwave, Millimeter and Submillimeter Waves (MSMW), 1 (Ukraine: Kharkiv: 2010).

21. J.M. Vigoureux, J. Opt. Soc. Am. A 8, 1697 (1991). 